\title{
Water birth: is the water an additional reservoir for group B streptococcus?
}

Received: 6 July 2005 / Accepted: 12 July 2005/Published online: 6 October 2005

(C) Springer-Verlag 2005

\begin{abstract}
Objective: Water birth became popular in the last years, despite the fact that many questions like the risk of infection for the newborn remain unanswered. Group B streptococcal (GBS) infections in the newborn remain a challenge in obstetrics and neonatology. Method: We conducted a prospective trial to study the impact of water birth on the colonization rate of the bath water and, more importantly, the GBS-colonization rate of the newborn. Result: After water birth the bath water was significantly more often colonized with GBS than after immersion followed by a delivery in bed. The newborns, however, showed no difference in GBS colonization and there was even a trend towards less GBS colonization of the newborn after a water delivery. Conclusion: Regarding GBS colonization of the newborn during water birth there might be a wash out effect, which protects the children during the delivery.
\end{abstract}

Keywords Immersion - Water delivery - Group B streptococcus

\section{Introduction}

Since Odent [9] published the first 100 cases of a so called "birth under water", this method of childbirth became more and more popular and is offered in many

The science fund of the University Hospital Basel, Switzerland, provided financial support. There are no conflicts of interest to declare

R. Zanetti-Dällenbach $(\bowtie) \cdot$ O. Lapaire $\cdot$ A. Maertens

W. Holzgreve · I. Hösli

University Women's Hospital Basel, Spitalstrasse 21,

4031 Basel, Switzerland

E-mail: rzanetti@uhbs.ch

Tel.: + 41-61-2659017

Fax: + 41-61-2659198

R. Frei

Bacteriology Laboratory, University Hospitals,

Basel, Switzerland hospitals, although there are few prospective randomized studies available $[2,5,10,14]$.

Infections due to group B streptococcus (GBS) (Streptococcus agalactiae) are common neonatal infections and can have great impact on a child's life as well as the family and society [13]. There are no data concerning GBS-positive women who wish to deliver their babies in the bathtub. In theory it could be possible that GBS colonized water during water delivery might be an additional reservoir for a GBS acquisition of the newborn $[1,7]$.

\section{Materials and methods}

Since March 1998 we offered water birth to all low-risk pregnant women. The women were informed about this birthing-method and our guidelines concerning water birth. Those who were interested in water birth signed a written consent. To protect the staff from infections, all interested women had to agree to be tested negative on HIV, hepatitis $\mathrm{B}$ and $\mathrm{C}$ before being admitted into the study $[4,11,12]$. The study was approved by the local ethical committee.

From March 1998 until May 2002 a total of 521 women showed interest in having a water birth. In the same time period 6,800 women gave birth at our institution. From all women interested in having a water birth eight had to be excluded. From the remaining 513 women $89(17.3 \%)$ had a water birth and composed the study group; $133(25.9 \%)$ had a relaxation bath (immersion in water) followed by a spontaneous cephalic delivery and composed the control group. All other women were not subject of this study: $146(28.5 \%)$ had a spontaneous cephalic delivery without immersion and $145(28.3 \%)$ experienced an operative vaginal delivery or a cesarean section.

Between the 37th and 40th week of gestation we took both vaginal and rectal swabs from all women to check for their GBS carrier-status. Additionally we analyzed water samples (1 1$)$ for GBS. We collected the water 
samples after the women left the bathtub. Within the first hour after delivery we took both nasal and pharyngeal swabs from the neonate, to check whether the children were colonized by GBS during delivery. Because we did not look at an external colonization we abstained from swabs taken from the meatus acusticus externus and the umbilical cord. In 64 cases of the study group and in 46 cases of the control group we were allowed to take swabs from the neonate. Therefore, in 64 cases from the 89 women in the study group and in 46 cases from the 133 women in the control group we had complete results.

All specimens were inoculated into a highly sensitive selective enrichment broth (Todd Hewitt supplemented with $10 \mathrm{mg} / 1$ colistine and $15 \mathrm{mg} / 1$ nalidixic acid). GBS was detected in the broth cultures by hybridization with a commercially available gene probe (AccuProbe ${ }^{\mathrm{TM}}$ ). GBS-positive women received intrapartum chemoprophylaxis.

The bathtubs are first cleaned with soap and afterwards disinfected with Kohrsolin FF.

\section{Result}

The laboring women in the two groups were comparable concerning origin, occupation, maternal age and week of gestation. There was no difference in APGAR, weight, arterial $\mathrm{pH}$ in the neonates in the two groups.

Twenty $(20 / 64 ; 31.25 \%)$ women were GBS carriers in the study group and $16(16 / 46 ; 34.8 \%)$ in the control group (ns, $P=0.854$ ). From the study/control group $13 /$ 11 women had positive rectal and vaginal cultures, $0 / 2$ positive vaginal and negative rectal cultures and $7 / 3$ negative vaginal and positive rectal cultures.

The cultures of the water samples were positive in 13 $(13 / 20 ; 65 \%)$ cases in the study group and in $4(4 / 16$; $25 \%)$ in the control group $(P=0.022)$.

In three cases $(3 / 20 ; 15 \%)$ nasal and pharyngeal swabs were positive in the study group and in five cases $(5 / 16 ; 31 \%)$ in the control group (ns; $P=0.421)$. In the study/control group $1 / 2$ children had positive nasal and pharyngeal cultures, $1 / 1$ child had positive pharyngeal and negative nasal cultures and $1 / 2$ children had negative pharyngeal and positive nasal cultures (Table 1).

Statistics: two tailed Fischer exact test for $2 \times 2$ tables.

\section{Discussion}

Clinical GBS infections of the children were not observed in both groups [3]. The likelihood of invasive disease was minimal due to the small sample size. There was also no statistical difference in the number of GBScarriers in the two groups. After water delivery the water was significantly more often colonized with GBS than after immersion.

Even though the water might be an additional reservoir for GBS acquisition for the newborn, our data showed, however, that there was not a statistically significant difference concerning the colonization of GBS of the newborn in the study and control group. The results even showed a trend towards less GBS colonization of the newborn after a water birth [8].

We therefore feel justified, even if the sample size is small, to conclude that water birth is safe also for GBS carriers concerning the bacterial colonization rate of newborns.

A potential explanation for our findings might be that during water delivery there could be a "wash out effect", which protects a newborn from GBS colonization.

\section{Summary}

Since the first publication in The Lancet (1983) about water deliveries, this mode of delivery became popular in some parts of the industrialized world. Studies, which confirm or refute the safety of this mode of delivery, are still lacking. Infections, especially GBS infections in the newborn remain a problem in daily obstetric and neonatal practice. The preliminary data of our prospective trial show for the first time that after water deliveries the bath water was significantly more often contaminated with GBS than in the control group after immersion followed by a delivery in bed. This might indicate that the contaminated bath water could be an additional reservoir for GBS acquisition for the newborn. The data of the newborns showed that there was no significant difference concerning the acquisition of GBS in the study and control group. The results showed a trend towards less GBS acquisition of the newborn after a water delivery.

\begin{tabular}{|c|c|c|c|c|c|}
\hline & \multicolumn{2}{|c|}{ Study group } & \multicolumn{2}{|c|}{ Control group } & $P$ value \\
\hline GBS pos & 20 & 31.25 & 16 & 34.8 & 0.85 \\
\hline Contaminated water & 13 & 65 & 4 & 25 & 0.002 \\
\hline GBS contamination of the children & 3 & 19 & 5 & 31 & 0.42 \\
\hline
\end{tabular}




\section{References}

1. Alderdice F, Renfrew M, Garcia J, McCandlish R (1993) Labour and birth in water. Lancet 342:1563

2. Alderdice F, Renfrew M, Marchant S, Ashurst H, Hughes P, Gerridge G, Garcia J (1995) Labour and birth in water in England and Wales. BMJ 310:837

3. Bowden K, Kessler D, Pinette M, Wilson E (2003) Underwater birth: missing the evidence or missing the point? Pediatrics 112(4):972-973

4. Brocklehurst P, Garcia J, Lumley J (1996) Birthing pools and infection control. Lancet 348:275

5. Cluett E, Pickering R, Getliffe K, Saunders N (2004) Randomized controlled trial of labouring in water compared with standard of augmentation for management of dystocia in first stage of labour. BMJ. DOI 10.1136/bmj.37963.606412EE

6. Geissbühler V, Stein S, Eberhard J (2004) Waterbirths compared with landbirths: an observational study of nine years. J Perinat Med 32:308-314
7. Gilbert R, Tookey P (1999) Perinatal mortality and morbidity among babies delivered in water: surveillance study and postal survey. BMJ 319:483-487

8. Johnson P (1996) Birth under water - to breathe or not to breathe. BJOG 103:202-208

9. Odent M (1983) Birth under water. Lancet 24/31:1476-1477

10. Otigbah C, Dhanjal M, Harmsworth G, Chard T (2000) A retrospective comparison of water births and conventional vaginal deliveries. Eur J Obstet Gynecol Reprod Biol 91:15-20

11. Ridgway GL, Tedder RS (1996) Birthing pools and infection control. BMJ 347:1051-1052

12. Roome A, Spencer R (1996) Birthing pools and infection control. Lancet 348:274

13. Schuchat A (2000) Neonatal group B streptococcal disease-screening and prevention. NEJM 343:209-210

14. Woodward J, Kelly S (2004) A pilot study for a randomized controlled trial of waterbirth versus land birth. BJOG 111:537545 